\title{
ALGORITHM FOR RELATIONAL DATABASE NORMALIZATION UP TO 3NF
}

\author{
Moussa Demba \\ Department of Computer Science \& Information, Aljouf University \\ Sakaka, Kingdom of Saudi Arabia \\ bah.demba@ju.edu.sa
}

\begin{abstract}
When an attempt is made to modify tables that have not been sufficiently normalized undesirable sideeffects may follow. This can be further specified as an update, insertion or deletion anomaly depending on whether the action that causes the error is a row update, insertion or deletion respectively. If a relation $R$ has more than one key, each key is referred to as a candidate key of $R$. Most of the practical recent works on database normalization use a restricted definition of normal forms where only the primary key (an arbitrary chosen key) is taken into account and ignoring the rest of candidate keys.

In this paper, we propose an algorithmic approach for database normalization up to third normal form by taking into account all candidate keys, including the primary key. The effectiveness of the proposed approach is evaluated on many real world examples.
\end{abstract}

\section{KEYWORDS}

Relational database, Normalization, Normal forms, functional dependency, redundancy.

\section{INTRODUCTION}

Normalization is, in relational database design, the process of organizing data to minimize redundancy. It usually involves dividing a database into two or more tables and defining relationships between the tables. The objective is to isolate data so that additions, deletions, and modifications of a field can be made in just one table and then propagated through the rest of the database via the defined relationships. Edgar Codd, the inventor of the relational model, also introduced the concept of normalization and Normal Forms (NF). The normal forms of relational database theory provide criteria for determining a table's degree of vulnerability to logical inconsistencies and anomalies. The higher the normal form applicable to a table, the less vulnerable it is. In general, normalization requires additional tables and some designers find this first difficult and then cumbersome.

Violating one of the first three rules of normalization, make the application anticipates any problems that could occur, such as redundant data and inconsistent dependencies.

When using the general definitions of the second and third normal forms (2NF and 3NF for short) we must be aware of partial and transitive dependencies on all candidate keys and not just the primary key. This can make the process of normalization more complex; however, the general definitions place additional constraints on the relations and may identify hidden redundancy in relations that could be missed [1].

A functional dependency $\mathrm{X} \rightarrow \mathrm{A}$ is partial if some attribute $\mathrm{B} \in \mathrm{X}$ can be removed from $\mathrm{X}$ and the dependency still holds. Let $\mathrm{A}, \mathrm{B}$, and $\mathrm{C}$ be attributes of a relation $\mathrm{R}, \mathrm{A} \rightarrow \mathrm{B}$ and $\mathrm{B} \rightarrow \mathrm{C}$ be two

DOI : $10.5121 /$ ijdms.2013.5303 
dependencies that hold in $\mathrm{R}$. Then $\mathrm{C}$ is transitively dependent on $\mathrm{A}$ via $\mathrm{B}$ (provided that $\mathrm{A}$ is not functionally dependent on B or C) [1].

Most of the recent works on database normalization are web-based tools without presenting algorithms and define the two notions, $2 \mathrm{NF}$ and $3 \mathrm{NF}$, with respect to primary keys only, ignoring the other candidate keys as in [2], [3, [4], [5]. However, the original definitions of the two notions as given in [6], [7] , [1], [8] consider all candidate keys. To see the difference between the two approaches, suppose we have a schema relation $\mathrm{R}(\mathrm{A}, \mathrm{B}, \mathrm{C}, \mathrm{D}, \mathrm{E}, \mathrm{F})$ together with the dependencies $\mathrm{F}=\{\mathrm{A} \rightarrow \mathrm{BCDEF} ; \mathrm{BC} \rightarrow \mathrm{ADEF} ; \mathrm{B} \rightarrow \mathrm{D} ; \mathrm{E} \rightarrow \mathrm{F}\}$

In this example, we have two candidate keys $A$ and $B C$. If $A$ is selected as the primary key of the relation, and ignoring $\mathrm{BC}$ then there is no partial dependencies on $\mathrm{A}$, implying that the relation is in 2NF. But if all candidate keys are taken into account (that is the general definition is considered), although there are no partial dependencies on A, we have a partial dependency $\mathrm{B} \rightarrow \mathrm{D}$ on candidate key $B C$, implying that the relation is not in $2 N F$. This make normalization process particularly confusing for many designers. In this paper, we propose an algorithmic approach for database normalization that uses the original and general definitions of normal forms. The general definitions take into account all candidate keys of a schema relation. For each synthesized relation, a primary key is also generated. The algorithms are presented step-by-step so designers can learn and implement them easily. Hereafter, all input schema relations are supposed at least in first normal form (1NF).

Throughout the paper, $\mathrm{R}$ represents a relational schema; $A, B, C, \ldots$ denote attributes; $X, Y$ and $Z$ denote set of attributes, and $\mathrm{F}$ a set of functional dependencies.

The rest of the paper is organized as follows: section 1 presents some basic concepts and notations, in section 2 we present a procedure for removing redundant attributes, in section 3 we present an algorithm for removing redundant attributes and another for redundant dependencies. Section 4 presents an algorithm for classifying dependencies into full and partial and in section 5 we present the algorithms for the decomposition into $2 \mathrm{NF}$ and 3NF respectively. In section 6 a complete and practice example is presented and in section 7 we conclude the paper.

\section{REMOVING REDUNDANT ATTRIBUTES AND DEPENDENCIES}

Before determining partial dependencies, some redundant functional dependencies could be removed. To do that, many algorithms have been proposed for removing redundant dependencies, called minimal cover [9], [10]. To achieve this goal, one needs to compute the closure of a given set of attributes and then remove redundant attributes.

\section{Definition 1.}

Given a relation $R$, a set of attributes $X$ in $R$ is said to functionally determine another set of attributes $Y$, also in $R$, written $X \rightarrow Y$, if and only if each $X$ value is associated with precisely one $Y$ value; $R$ is then said to satisfy the functional dependency $X \rightarrow Y$.

\section{Definition 2.}

A set of functional dependencies $F$ is in canonical form if each functional dependency $\mathrm{X} \rightarrow \mathrm{A}$ in $F, A$ is a singleton attribute.

Hereafter, all functional dependencies are supposed in canonical form. 
International Journal of Database Management Systems ( IJDMS ) Vol.5, No.3, June 2013

Let $\mathrm{X}$ be a set attributes and $\mathrm{F}$ be a set of functional dependencies. Let $\mathrm{X}^{+}$be the set of all attributes that depend on a subset of $\mathrm{X}$ with respect to $\mathrm{F}$, i.e., $\mathrm{X}^{+}$is the set of attributes $\mathrm{Z}$, such that $\mathrm{X} \rightarrow \mathrm{Z} \in \mathrm{F}$. $\mathrm{X}^{+}$is called the closure of $\mathrm{X}$ w.r.t $\mathrm{F}$.

The Algorithm Al computes the closure of a given set of attributes w.r.t F:

Algorithm A1: computes $\mathrm{X}^{+}$.

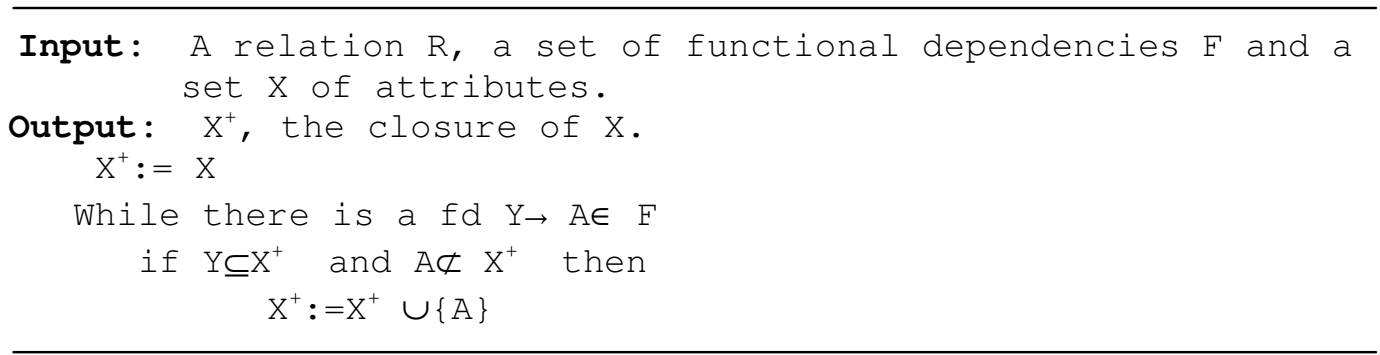

To check if a functional dependency $\mathrm{Y} \rightarrow \mathrm{A}$ holds in a set of dependencies $\mathrm{F}$, we compute the closure of $\mathrm{Y}$ and check If $\mathrm{A} \subseteq \mathrm{Y}^{+}$. This test is particularly useful, as we will see later in the next algorithm.

We can use attribute closure to remove redundant dependencies from a set of functional dependencies. We can do this by examining each dependency in turn to see if it is redundant. A dependency is redundant if it can be inferred from the other dependencies, and can thus be removed.

Given a set of dependencies $\mathrm{F}$, an attribute $\mathrm{B}$ is extraneous in $\mathrm{X} \rightarrow \mathrm{A}$ with respect to $\mathrm{F}$ if $\mathrm{A} \in(\mathrm{X}$ $\mathrm{B})^{+}$. If $\mathrm{B} \in(\mathrm{X}-\mathrm{B})^{+}$then $\mathrm{B}$ is called an implied extraneous attribute. If $\mathrm{B}$ is extraneous, but not implied, then it is nonimplied. For example, suppose we have the functional dependencies $\mathrm{A}, \mathrm{B} \rightarrow \mathrm{C}$ and $\mathrm{A} \rightarrow \mathrm{C}$ in $\mathrm{F}$. Then $\mathrm{B}$ is a nonimplied extraneous attribute in $\mathrm{A}, \mathrm{B} \rightarrow \mathrm{C}$. As another example, suppose we have the dependencies $\mathrm{A}, \mathrm{B} \rightarrow \mathrm{C}$ and $\mathrm{A} \rightarrow \mathrm{B}$ in $\mathrm{F}$. Then $\mathrm{B}$ is an implied extraneous attribute in $\mathrm{A}, \mathrm{B} \rightarrow \mathrm{C}$.

$\mathrm{F}$ is called partially left-reduced if no attributes are implied extraneous attribute. The elimination of non-implied extraneous attribute is postponed until the time that redundant dependencies are eliminated, using the Algorithm A3. Many algorithms, sometimes difficult to reuse, have been proposed in [11], [12] for removing extraneous attributes.

In our approach, we propose the following algorithm to eliminate implied extraneous attributes by minimizing attribute closure computations. Algorithm $A 3$ removes any implied extraneous attributes. Our observation is that any implied extraneous attribute has to appear both on the lefthand side of at least one dependency and on the right-hand side of at least one dependency. Therefore, categorizing attributes according to their appearance on the left-hand side and righthand side of dependencies could reduce the number of attributes closure computation.We define the sets:

$$
\begin{aligned}
& \mathrm{ro}=\{\mathrm{A} / \mathrm{A} \text { occurs only on the right hand side of dependencies in } \mathrm{F}\} \\
& \mathrm{l}=\{\mathrm{A} / \mathrm{A} \text { occurs only on the left hand side of dependencies in } \mathrm{F}\} \\
& \mathrm{l}=\{\mathrm{A} / \mathrm{A} \notin \mathrm{ro} \text { and } \mathrm{A} \notin \mathrm{lo}\}
\end{aligned}
$$

Note that lonro= $\phi$, lonlr= $\phi$ and $r o n l r=\phi$. Moreover, the set $l o$, if not empty, contains keyattributes. 
Lemma 1. If $B$ is an implied extraneous attribute in a set of dependencies $F$, then $B \in l r$.

Proof. It is easy to see that an implied extraneous attribute, as defined above, has to occur on the left-hand side of a dependency, then cannot be an element of the set ro.

Lemma 2. If $\mathrm{B}$ is an extraneous implied attribute, then $\mathrm{B} \notin l o$ and $\mathrm{B} \notin r o$.

In contrast to the algorithm proposed in [12] that tests the redundancy of all attributes in $(l o \cup l r)$, we will test only for the attributes in $l r$. Let IXI denotes the cardinality (number of attributes) of X.

Algorithm A2: Removes implied extraneous attributes.

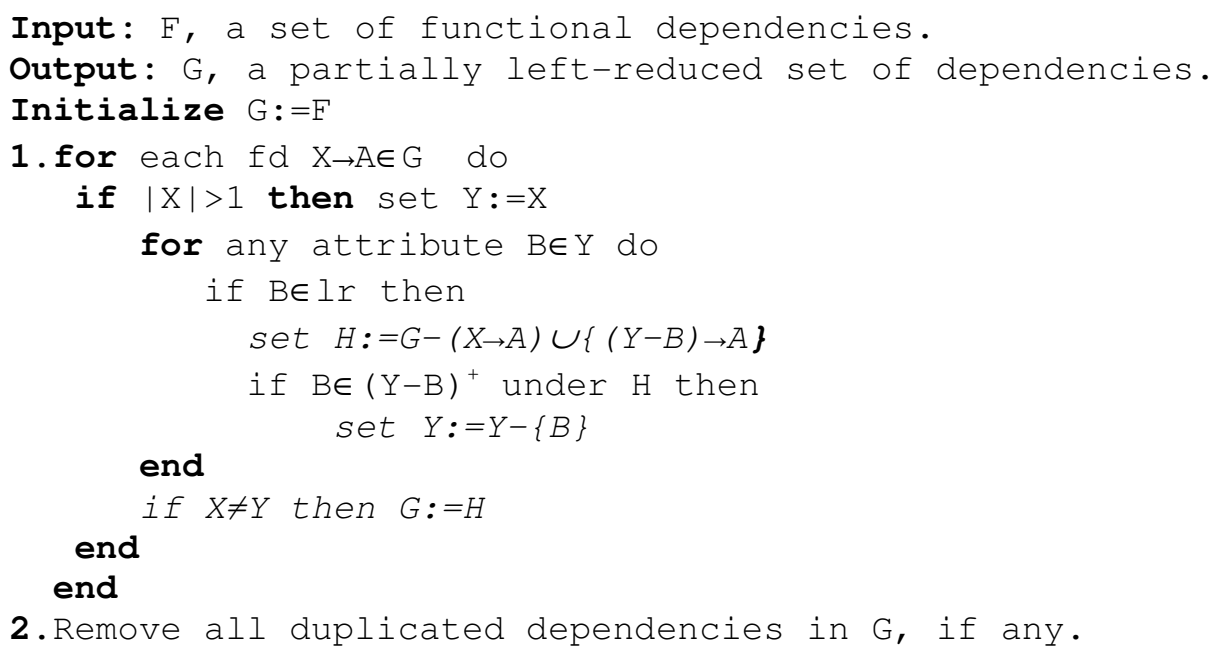

This algorithm is substantially better than the one proposed in [12].

Example 1 [12]: Let $\mathrm{F}$ be the set of dependencies:

$$
\begin{aligned}
& \mathrm{AB} \rightarrow \mathrm{D} ; \mathrm{B} \rightarrow \mathrm{A} ; \mathrm{ABC} \rightarrow \mathrm{D} ; \mathrm{D} \rightarrow \mathrm{A} ; \mathrm{AB} \rightarrow \mathrm{E} ; \mathrm{B} \rightarrow \mathrm{H} ; \mathrm{ABC} \rightarrow \mathrm{J} ; \mathrm{AB} \rightarrow \mathrm{F} ; \\
& \mathrm{B} \rightarrow \mathrm{G} ; \mathrm{ABC} \rightarrow \mathrm{K} ; \mathrm{BN} \rightarrow \mathrm{H} ; \mathrm{AB} \rightarrow \mathrm{G} \\
& \mathrm{G}:=\mathrm{F}, \mathrm{lr}=\{\mathrm{A}, \mathrm{D}\}, \mathrm{lo}=\{\mathrm{B}, \mathrm{C}\} \text { and } \mathrm{ro}=\{\mathrm{E}, \mathrm{F}, \mathrm{G}, \mathrm{H} . J, \mathrm{~K}\}
\end{aligned}
$$

According to lemma 2, there is no need to check for the attributes in $l o$ or in ro.

-For $\mathbf{A B} \rightarrow \mathbf{D}$.

$B$ cannot be an implied extraneous attribute as $B \notin l r$, then we check for $A$ : we have $\mathrm{A} \in(\mathrm{Y}-\mathrm{A})^{+} ; \mathrm{Y}=\mathrm{Y}-\mathrm{A}$

$G$ is changed by replacing $A B \rightarrow D$ by $B \rightarrow D$ :

$$
\begin{aligned}
& \mathrm{B} \rightarrow \mathrm{D} ; \mathrm{B} \rightarrow \mathrm{A} ; \mathrm{BC} \rightarrow \mathrm{D} ; \mathrm{D} \rightarrow \mathrm{A} ; \mathrm{B} \rightarrow \mathrm{E} ; \mathrm{B} \rightarrow \mathrm{H} ; \mathrm{BC} \rightarrow \mathrm{J} ; \mathrm{B} \rightarrow \mathrm{F} ; \mathrm{B} \rightarrow \mathrm{G} ; \\
& \mathrm{BC} \rightarrow \mathrm{K} ; \mathrm{BN} \rightarrow \mathrm{H} ; \mathrm{B} \rightarrow \mathrm{G}
\end{aligned}
$$

\section{-For $\mathbf{B C} \rightarrow \mathbf{D}$.}

$G$ will not change as $B, C \notin l r$ 


\section{-For $\mathbf{B N} \rightarrow \mathbf{H}$.}

$G$ will not change as $B, N \notin l r$

$B \rightarrow G$ is duplicated, then has to be removed we get the partially left-reduced dependencies $F^{\prime}$ of $F$ :

$\mathrm{B} \rightarrow \mathrm{D} ; \mathrm{B} \rightarrow \mathrm{A} ; \mathrm{BC} \rightarrow \mathrm{D} ; \mathrm{D} \rightarrow \mathrm{A} ; \mathrm{B} \rightarrow \mathrm{E} ; \mathrm{B} \rightarrow \mathrm{H} ; \mathrm{BC} \rightarrow \mathrm{J} ; \mathrm{B} \rightarrow \mathrm{F} ; \mathrm{B} \rightarrow \mathrm{G} ; \mathrm{BC} \rightarrow \mathrm{K} ;$

$\mathrm{BN} \rightarrow \mathrm{H}$

There is no more extraneous implied attributes in $\mathrm{F}^{\prime}$, while the nonimplied extraneous attribute $\mathrm{N}$, it will be removed by the next procedure.

The next step consists to remove redundant dependencies. The main idea is to compare the lhs of dependencies that have the same rhs: if a dependency $\mathrm{f}_{1}: \mathrm{X} \rightarrow \mathrm{A}$ is redundant in $\mathrm{F}$, then there is at least a dependency $f_{2}: Y \rightarrow A$ in $F$ where $X \rightarrow Y \in F^{+}$. We write $X_{F}^{+}$to mean that the closure of $X$ w.r.t a set of dependencies F. Algorithmically this can be expressed as follows:

Algorithm A3: Removes redundant dependencies.

Input: $F$, a set of partially left-reduced dependencies.

Output: $F_{m}$, a minimal cover of $\mathrm{F}$

1. $\mathrm{F}_{\mathrm{m}}:=\mathrm{F}$

2. for each $X \rightarrow A \in F_{m}$

while there exists a $Y \rightarrow A \in F_{m}$

$\mathrm{G}:=\mathrm{F}_{\mathrm{m}}-(\mathrm{Y} \rightarrow \mathrm{A})$

if $\mathrm{X} \subseteq \mathrm{Y}_{\mathrm{G}}^{+}$then

$$
\mathrm{F}_{\mathrm{m}}:=\mathrm{G}
$$

end

end

end

3. $\mathrm{F}_{\mathrm{m}}$ is the minimal cover of $\mathrm{F}$.

To illustrate the algorithm, let $\mathrm{F}$ be the dependencies given in example 1.

1. Initialization of $F_{m}$ to:

$\mathrm{B} \rightarrow \mathrm{D} ; \mathrm{B} \rightarrow \mathrm{A} ; \mathrm{BC} \rightarrow \mathrm{D} ; \mathrm{D} \rightarrow \mathrm{A} ; \mathrm{B} \rightarrow \mathrm{E} ; \mathrm{B} \rightarrow \mathrm{H} ; \mathrm{BC} \rightarrow \mathrm{J} ; \mathrm{B} \rightarrow \mathrm{F} ; \mathrm{B} \rightarrow \mathrm{G} ; \mathrm{BC} \rightarrow \mathrm{K} ;$

$\mathrm{BN} \rightarrow \mathrm{H}$

2. Summarized in table 1 .

3. $\mathrm{F}_{\mathrm{m}}=\{\mathrm{B} \rightarrow \mathrm{D} ; \mathrm{B} \rightarrow \mathrm{E} ; \mathrm{B} \rightarrow \mathrm{F} ; \mathrm{B} \rightarrow \mathrm{H} ; \mathrm{B} \rightarrow \mathrm{G} ; \mathrm{BC} \rightarrow \mathrm{J} ; \mathrm{BC} \rightarrow \mathrm{K} ; \mathrm{D} \rightarrow \mathrm{A}\}$ 
International Journal of Database Management Systems ( IJDMS ) Vol.5, No.3, June 2013

Table 1. Sketch elimination of redundant dependencies.

\begin{tabular}{|c|l|l|l|}
\hline Pass & $\mathbf{X} \rightarrow \mathbf{A}$ & \multicolumn{1}{|l|}{$\rightarrow \mathbf{A}$} & \multicolumn{1}{|c|}{$\mathbf{F}_{\mathbf{m}}$} \\
\hline 1 & $\mathrm{~B} \rightarrow \mathrm{D}$ & $\mathrm{BC} \rightarrow \mathrm{D}, \mathrm{B} \in(\mathrm{BC})^{+}$ & $\mathrm{F}_{\mathrm{m}}:=\mathrm{F}_{\mathrm{m}}-(\mathrm{BC} \rightarrow \mathrm{D})$ \\
\hline 2 & $\mathrm{~B} \rightarrow \mathrm{E}$ & & \\
\hline 3 & $\mathrm{~B} \rightarrow \mathrm{F}$ & & \\
\hline 4 & $\mathrm{~B} \rightarrow \mathrm{A}$ & & $\mathrm{F}_{\mathrm{m}}:=\mathrm{F}_{\mathrm{m}}-(\mathrm{BN} \rightarrow \mathrm{H})$ \\
\hline 5 & $\mathrm{~B} \rightarrow \mathrm{H}$ & $\mathrm{BN} \rightarrow \mathrm{H}, \mathrm{B} \in(\mathrm{BN})^{+}$ & \\
\hline 6 & $\mathrm{~B} \rightarrow \mathrm{G}$ & & \\
\hline 7 & $\mathrm{BC} \rightarrow \mathrm{J}$ & & \\
\hline 8 & $\mathrm{BC} \rightarrow \mathrm{K}$ & & $\mathrm{F}_{\mathrm{m}}:=\mathrm{F}_{\mathrm{m}}-(\mathrm{B} \rightarrow \mathrm{A})$ \\
\hline 9 & $\mathrm{D} \rightarrow \mathrm{A}$ & $\mathrm{B} \rightarrow \mathrm{A}, \mathrm{D} \in \mathrm{B}^{+}$ & \\
\hline
\end{tabular}

In [12] they claimed that their (fast) algorithm computes 5 closures and the standard one 19 closures to remove redundant dependencies. As we can see in the table 1 , only 3 computations are needed using our Algorithm A3, i.e., passes 1, 5 and 9, that is a substantial improvement. Moreover, for the other non-redundant dependencies, no extra effort is needed.

Unfortunately, there can be several minimal covers for a set of functional dependencies. We can always find at least one minimal cover $\mathrm{F}_{\mathrm{m}}$ for any set of dependencies $\mathrm{F}$ using Algorithm A3.

Theorem 1. Let $\mathrm{F}$ be a set of partially left-reduced dependencies, and $\mathrm{F}_{\mathrm{m}}$ its minimal cover obtained by the Algorithm A3. Then we have $\mathrm{F}^{+} \equiv \mathrm{F}_{\mathrm{m}}{ }^{+}$.

\section{Full and Partial DePendencies}

A functional dependency $\mathrm{X} \rightarrow \mathrm{A}$ is a full functional dependency if removal of any attribute $\mathrm{B}$ from $X$ means that the dependency does not hold any more; that is, for any attribute $B \in X,(X-B)$ does not functionally determine $\mathrm{A}$. A functional dependency $\mathrm{X} \rightarrow \mathrm{A}$ is a partial dependency if $\mathrm{A}$ is not a key-attribute and some attribute $\mathrm{B} \in \mathrm{X}$ can be removed from $\mathrm{X}$ and the dependency still holds; that is, for some $\mathrm{B} \in \mathrm{X},(\mathrm{X}-\mathrm{B}) \rightarrow \mathrm{A}$ [7]. Full and partial dependencies are generated as follows:

Algorithm A4: Determines full and partial dependencies.

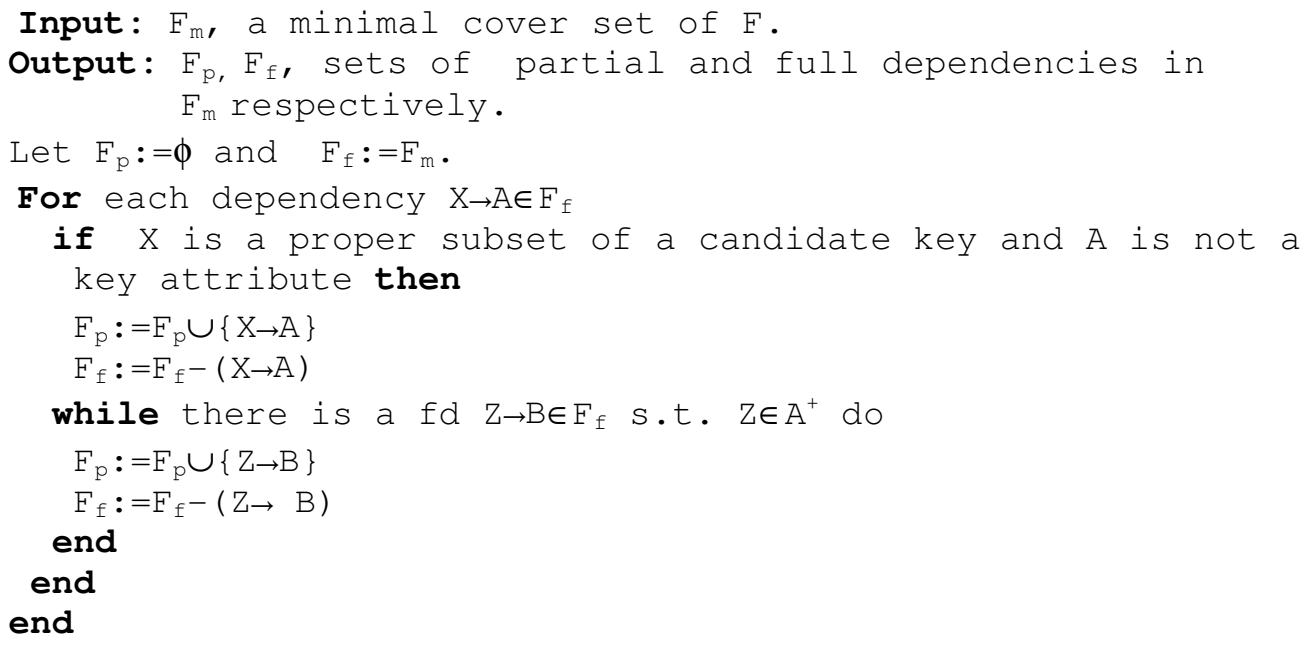


International Journal of Database Management Systems ( IJDMS ) Vol.5, No.3, June 2013

In the If-statement, we make condition on the candidate keys and not the primary key, because if a relation has many keys, all have to be considered (see the definition of $2 \mathrm{NF}$ in the next section).

We can continue our running example by initializing $F_{\mathrm{f}}$ to:

$$
\{\mathrm{B} \rightarrow \mathrm{D} ; \mathrm{B} \rightarrow \mathrm{F} ; \mathrm{B} \rightarrow \mathrm{G} ; \mathrm{D} \rightarrow \mathrm{A} ; \mathrm{B} \rightarrow \mathrm{E} ; \mathrm{B} \rightarrow \mathrm{H} ; \mathrm{BC} \rightarrow \mathrm{J} ; \mathrm{BC} \rightarrow \mathrm{K}\}
$$

we have one candidate key $\mathrm{BC}$, that is the primary key. $\mathrm{F}_{\mathrm{p}}=\phi$. For the lines $1-5, \mathrm{~B} \subseteq(\mathrm{BC})$, then we have partial dependencies.

Table 2. Sketch determination of $F_{f}$ and $F_{p}$.

\begin{tabular}{|l|l|l|}
\hline \multicolumn{1}{|c|}{$\mathbf{X} \rightarrow \mathbf{A}$} & \multicolumn{1}{|c|}{$\mathbf{F}_{\mathrm{f}}$} & \multicolumn{1}{c|}{$\mathbf{F}_{\mathrm{P}}$} \\
\hline $\mathrm{B} \rightarrow \mathrm{D}$ & $\mathrm{F}_{\mathrm{f}}:=\mathrm{F}_{\mathrm{f}}-\{\mathrm{B} \rightarrow \mathrm{D} ; \mathrm{D} \rightarrow \mathrm{A}\}$ & $\mathrm{F}_{\mathrm{P}}:=\mathrm{F}_{\mathrm{P}} \cup\{\mathrm{B} \rightarrow \mathrm{D} ; \mathrm{D} \rightarrow \mathrm{A}\}$ \\
\hline $\mathrm{B} \rightarrow \mathrm{E}$ & $\mathrm{F}_{\mathrm{f}}:=\mathrm{F}_{\mathrm{f}}-(\mathrm{B} \rightarrow \mathrm{E})$ & $\mathrm{F}_{\mathrm{P}}:=\mathrm{F}_{\mathrm{P}} \cup\{\mathrm{B} \rightarrow \mathrm{E}\}$ \\
\hline $\mathrm{B} \rightarrow \mathrm{F}$ & $\mathrm{F}_{\mathrm{f}}:=\mathrm{F}_{\mathrm{f}}-(\mathrm{B} \rightarrow \mathrm{F})$ & $\mathrm{F}_{\mathrm{P}}:=\mathrm{F}_{\mathrm{P}} \cup\{\mathrm{B} \rightarrow \mathrm{F}\}$ \\
\hline $\mathrm{B} \rightarrow \mathrm{H}$ & $\mathrm{F}_{\mathrm{f}}:=\mathrm{F}_{\mathrm{f}}-(\mathrm{B} \rightarrow \mathrm{H})$ & $\mathrm{F}_{\mathrm{P}}:=\mathrm{F}_{\mathrm{P}} \cup\{\mathrm{B} \rightarrow \mathrm{H}\}$ \\
\hline $\mathrm{B} \rightarrow \mathrm{G}$ & $\mathrm{F}_{\mathrm{f}}:=\mathrm{F}_{\mathrm{f}}-(\mathrm{B} \rightarrow \mathrm{G})$ & $\mathrm{F}_{\mathrm{P}}:=\mathrm{F}_{\mathrm{P}} \cup\{\mathrm{B} \rightarrow \mathrm{G}\}$ \\
\hline $\mathrm{BC} \rightarrow \mathrm{J}$ & & \\
\hline $\mathrm{BC} \rightarrow \mathrm{K}$ & & \\
\hline
\end{tabular}

The final result is:

$$
\begin{aligned}
& F_{f}=\{B C \rightarrow J ; B C \rightarrow K\} \\
& F_{P}=\{B \rightarrow D ; D \rightarrow A ; B \rightarrow E ; B \rightarrow F ; B \rightarrow G ; B \rightarrow H\}
\end{aligned}
$$

Lemma 3. $F_{m}=F_{f} \cup F_{P}$.

Lemma 4. Let $R$ be a relation schema and $F_{m}$ its minimal cover dependency set. If there is no partial dependency in $R$ then $F_{p}=\phi$.

\section{NORMALIZATION}

\subsection{The Second Normal Form}

A relation is in second normal form (abbreviated 2NF) if it is in 1NF and no non-key attribute is partially dependent on any candidate key [7], [1]. In other words, no $\mathrm{X} \rightarrow \mathrm{Y}$ where $\mathrm{X}$ is a strict subset of a candidate key and $\mathrm{Y}$ is a non-key attribute.

Simply, a table is in 2NF if and only if it is in $1 \mathrm{NF}$ and every non-key attribute of the relation is either dependent on the whole of any candidate key, or on another non-key attribute.

The following algorithm is used to decompose a $1 \mathrm{NF}$ relation schema $\mathrm{R}$ into $2 \mathrm{NF}$ :

Algorithm A5: Decomposes into $2 N F$.

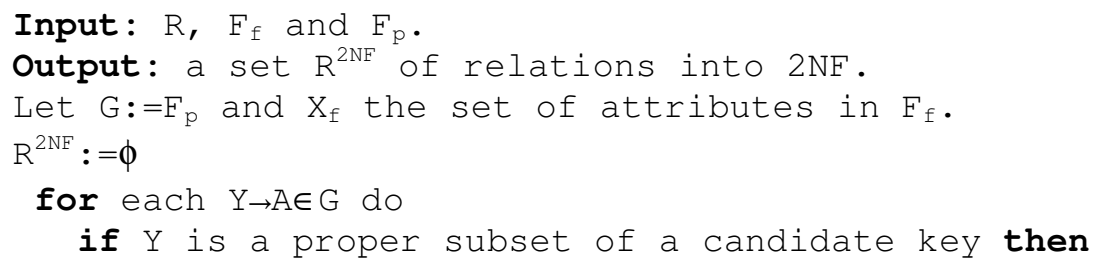




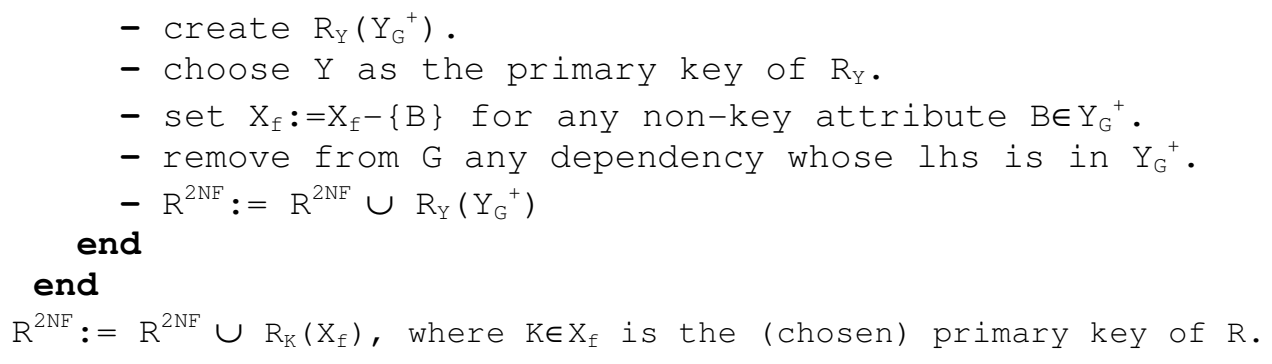

If $\mathrm{Y}$ is a key-attribute that means that $\mathrm{Y}$ is causing a partial dependency. The statement $X_{f}:=X_{f}$ $\{B\}$ is justified by the fact that $Y$ is already in $\mathrm{X}_{\mathrm{f}}$. Moreover, the resulting decomposition preserves the functional dependencies of $F_{m}$, because $Y \rightarrow A$ belongs to $R_{Y}$ and $Y \subseteq X_{f}$.

Theorem 2. Let $R$ be a $1 N F$ relation. We have the following results:

a. If $\mathrm{F}_{\mathrm{p}}=\phi$ then $R$ is automatically in $2 N F$.

b. If $\mathrm{F}_{\mathrm{p}} \neq \phi$ then $R$ is not in $2 N F$.

\section{Proof.}

a. If $\mathrm{F}_{\mathrm{p}}=\phi$, i.e., there is no partial dependency in $\mathrm{R}$, that means also that the first if-condition of the algorithm will never hold. Then $\mathrm{R}_{\mathrm{K}}$ is the only relation created by the Algorithm A5. As $R_{K}$ is partial dependency free (no partial dependency exists in $F_{f}$ ), then $R=R_{K}$ is in $2 N F$.

b. If $\mathrm{F}_{\mathrm{p}} \neq \phi$, i.e., the first if-condition of the algorithm holds at least once. Therefore, $\mathrm{R}$ is not in $2 N F$ as $\mathrm{R} \neq \mathrm{R}_{\mathrm{K}}$.

Let's assess our example:

$$
\begin{aligned}
& \mathrm{F}_{\mathrm{f}}=\{\mathrm{BC} \rightarrow \mathrm{J}, \mathrm{BC} \rightarrow \mathrm{K}\} \\
& \mathrm{F}_{\mathrm{p}}=\{\mathrm{B} \rightarrow \mathrm{D} ; \mathrm{D} \rightarrow \mathrm{A} ; \mathrm{B} \rightarrow \mathrm{E} ; \mathrm{B} \rightarrow \mathrm{F} ; \mathrm{B} \rightarrow \mathrm{G} ; \mathrm{B} \rightarrow \mathrm{H}\} \\
& \text { and } \mathrm{F}_{\mathrm{m}}=\mathrm{F}_{\mathrm{f}} \cup \mathrm{F}_{\mathrm{p}} .
\end{aligned}
$$

As we can see all attributes in $F_{f}$ depend fully on the only candidate key $\{B C\}$ and all the attributes in $\mathrm{F}_{\mathrm{p}}$ depend directly/indirectly on the key-attribute $\mathrm{B}$, i.e., $\mathrm{B}$ is causing a partial key. Therefore, the relations $R_{B C}$ and $R_{B}$ are created:

$$
\begin{aligned}
& \mathrm{R}_{\mathrm{BC}}(\underline{\mathrm{B}, \mathrm{C}}, \mathrm{J}, \mathrm{K}) \\
& \mathrm{R}_{\mathrm{B}}(\underline{\mathrm{B}}, \mathrm{D}, \mathrm{A}, \mathrm{E}, \mathrm{F}, \mathrm{G}, \mathrm{H})
\end{aligned}
$$

Note that when a $1 \mathrm{NF}$ relation has no composite candidate keys (candidate keys consisting of more than one attribute), the relation is automatically in $2 \mathrm{NF}$. If there is no composite candidate keys, then $\mathrm{F}_{\mathrm{p}}=\phi$ and by theorem 2 the relation is in $2 \mathrm{NF}$.

\subsection{Third Normal Form}

A relation is in third normal form (abbreviated $3 \mathrm{NF}$ ) if it is in $2 \mathrm{NF}$ and none of its non-key attributes are transitively dependent upon any candidate key [6], [1], [7]. An alternative (simpler) definition is a relation is in $3 N F$ if in every non-trivial dependency $\mathrm{X} \rightarrow \mathrm{A}$ either $\mathrm{X}$ is a super key or A is a key attribute (i.e., A is contained within a candidate key).

The transitive dependencies set on $2 \mathrm{NF}$ relations is defined by: 
International Journal of Database Management Systems ( IJDMS ) Vol.5, No.3, June 2013

$F_{t}=\left\{X \rightarrow A \in F_{m}\right\}$ such that

i. $\quad X$ is not a candidate key, and

ii. $\quad A$ is not a key attribute.

Note that partial dependencies violate the 3NF. We have the following characterization.

Theorem : A relation schema $\mathrm{R}$ is in third normal form if and only if for every key $\mathrm{K}$ of $\mathrm{R}$ and every nonkey-attribute A depends directly on $\mathrm{K}$.

The following procedure is used to decompose a $1 \mathrm{NF}$ relation into $3 \mathrm{NF}$.

Algorithm A6. Decomposes into 3NF.

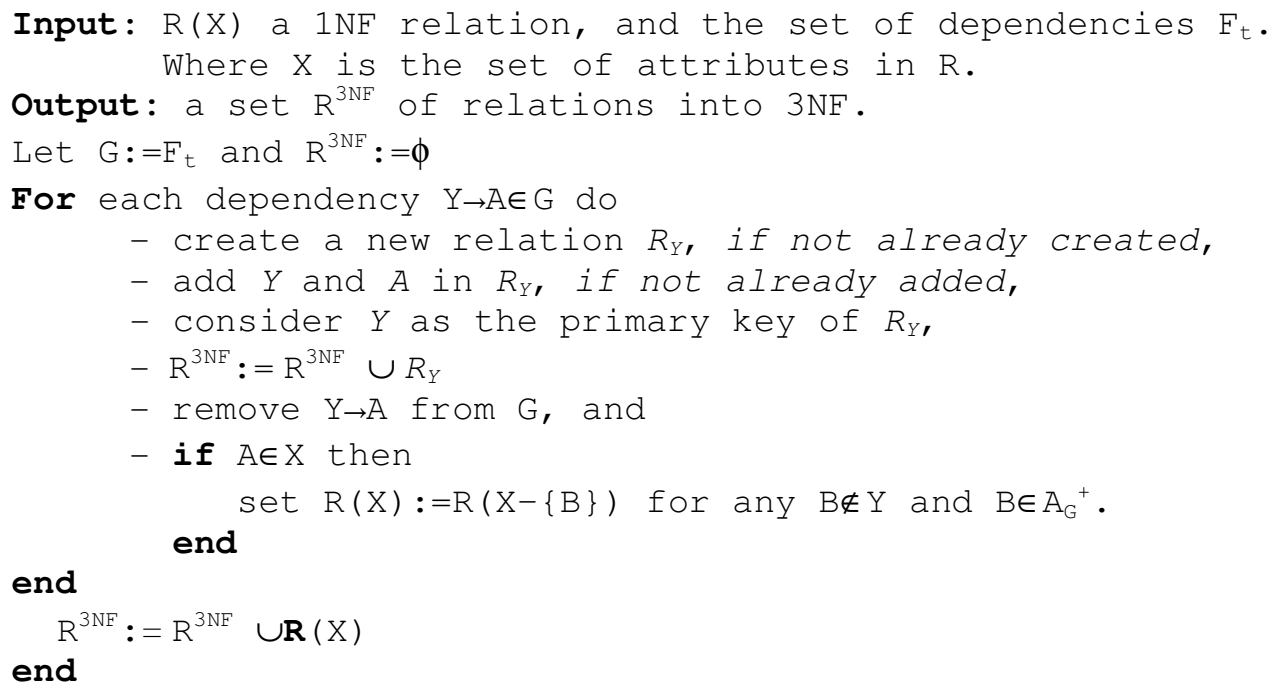

Lemma 5. If $F_{t} \neq \phi$, then the input relation $R$ is not in $3 N F$.

Now, we can complete our example given the initial $1 \mathrm{NF}$ relation with $\mathrm{BC}$ the only candidate key $\mathrm{BC}$ :

$\mathrm{R}_{\mathrm{BC}}(\mathrm{A}, \underline{\mathrm{B}, \mathrm{C}}, \mathrm{D}, \mathrm{E}, \mathrm{F}, \mathrm{G}, \mathrm{H}, \mathrm{J}, \mathrm{K})$

together with the following dependencies:

$$
\begin{aligned}
& \mathrm{F}_{\mathrm{m}}=\{\mathrm{BC} \rightarrow \mathrm{J} ; \mathrm{BC} \rightarrow \mathrm{K} ; \mathrm{B} \rightarrow \mathrm{D} ; \mathrm{D} \rightarrow \mathrm{A} ; \mathrm{B} \rightarrow \mathrm{E} ; \mathrm{B} \rightarrow \mathrm{F} ; \mathrm{B} \rightarrow \mathrm{G} ; \mathrm{B} \rightarrow \mathrm{H}\} \\
& \mathrm{F}_{\mathrm{t}}=\{\mathrm{B} \rightarrow \mathrm{D} ; \mathrm{D} \rightarrow \mathrm{A} ; \mathrm{B} \rightarrow \mathrm{E} ; \mathrm{B} \rightarrow \mathrm{F} ; \mathrm{B} \rightarrow \mathrm{G} ; \mathrm{B} \rightarrow \mathrm{H}\}
\end{aligned}
$$

According to the lemma $5, \mathrm{R}_{\mathrm{BC}}(\mathrm{X})$, where $X$ is the set of attributes, is not in $3 \mathrm{NF}$. Therefore new relations $\mathrm{R}_{\mathrm{B}}(\underline{B}, \mathrm{D}, \mathrm{E}, \mathrm{F}, \mathrm{G}, \mathrm{H})$ and $\mathrm{R}_{\mathrm{D}}(\underline{\mathrm{D}}, \mathrm{A})$ are then created and the attributes $\mathrm{D}, \mathrm{E}, \mathrm{F}, \mathrm{G}, \mathrm{H}$ are removed from $X$ because they are in $\mathrm{X}^{+}$. We get the final relations into 3NF:

$$
\begin{aligned}
& \mathrm{R}_{\mathrm{BC}}(\underline{B}, \mathrm{C}, \mathrm{J}, \mathrm{K}) \\
& \mathrm{R}_{\mathrm{B}}(\underline{\mathrm{B}}, \mathrm{D}, \mathrm{E}, \mathrm{F}, \mathrm{G}, \mathrm{H}) \\
& \mathrm{R}_{\mathrm{D}}(\underline{\mathrm{D}}, \mathrm{A})
\end{aligned}
$$


Example 2: Consider the schema relation

$\mathrm{R}($ isbn, invno, title, authors, publ, place, year)

together with the set of dependencies:

$\mathrm{F}_{\mathrm{m}}=\{($ title, authors, publ, place, year $) \rightarrow$ isbn; isbn $\rightarrow$ title; isbn $\rightarrow$ authors;

isbn $\rightarrow$ publ; isbn $\rightarrow$ place; isbn $\rightarrow$ year; invno $\rightarrow$ isbn $\}$

The attribute invno is the unique key of $\mathrm{R}$. The set of partial dependencies $\mathrm{F}_{\mathrm{p}}=\phi$ and the set of transitive dependencies :

$\mathrm{F}_{\mathrm{t}}=\{($ title, authors, publ, place, year $) \rightarrow$ isbn; isbn $\rightarrow$ title; isbn $\rightarrow$ authors;

isbn $\rightarrow$ publ; isbn $\rightarrow$ place; isbn $\rightarrow$ year $\}$

Therefore the relation $R$ is in $2 \mathrm{NF}$ but not in $3 \mathrm{NF}$ because $\mathrm{F}_{\mathrm{t}} \neq \phi$. R can be easily normalized by decomposition using the algorithm A6 as follows:

$\mathrm{R}_{1}$ ( title, authors, publ, place, year, isbn)

$\mathrm{R}_{2}$ (invno, title, authors, publ, place, year)

\section{A Complete Example}

To demonstrate the applicability of the above algorithms, consider the more realistic example presented in [1].

Suppose we want to normalize the $1 N F$ relation ClientRental up to $3 N F$. This relation contains 9 attributes and 17 dependencies:

Clientrental (clientNo, propertyNo, clientName, pAddress, rentStart, rentFinish, rent, ownerNo, ownerName)

with the following dependencies F:

$\begin{array}{lll}f_{1}: & \text { propertyNo,rentStart } & \rightarrow \text { rentFinish } \\ f_{2}: & \text { clNo,propertyNo } & \rightarrow \text { rentFinish } \\ f_{3}: & \text { clNo,rentStart } & \rightarrow \text { rentFinish } \\ f_{4}: \quad \text { clNo } & \rightarrow \text { clName } \\ f_{5}: \quad \text { propertyNo,rentStart } & \rightarrow \text { clName } \\ f_{6}: \quad \text { clNo,propertyNo } & \rightarrow \text { rentStart } \\ f_{7}: \quad \text { ownerNo } & \rightarrow \text { oName } \\ f_{8}: \quad \text { propertyNo } & \rightarrow \text { oName } \\ f_{9}: \quad \text { clNo,rentStart } & \rightarrow \text { oName } \\ f_{10}: \quad \text { clNo,rentStart } & \rightarrow \text { pAddress } \\ f_{11}: \quad \text { propertyNo } & \rightarrow \text { pAddress } \\ f_{12}: \quad \text { propertyNo } & \rightarrow \text { rent } \\ f_{13}: \quad \text { clNo,rentStart } & \rightarrow \text { rent } \\ f_{14}: \quad \text { propertyNo } & \rightarrow \text { ownerNo } \\ f_{15}: \quad \text { clNo,rentStart } & \rightarrow \text { ownerNo } \\ f_{16}: \quad \text { clNo,rentStart } & \rightarrow \text { propertyNo } \\ f_{17}: \quad \text { propertyNo,rentStart } & \rightarrow \text { clNo }\end{array}$


Step 1: First of all, we call the Algorithm $A 3$ to generate a minimal cover $F_{m}$ of $F$, as there is no implied extraneous attributes. Let $\operatorname{lhs}\left(f_{\mathrm{j}}\right) \subseteq \operatorname{lhs}\left(f_{\mathrm{i}}\right)^{+}$means that the left-hand-side of the dependency $f_{\mathrm{j}}$ is in the closure of the left-hand-side of the dependency $f_{\mathrm{i}}$, i.e. $\operatorname{lhs}\left(f_{i}\right) \rightarrow \operatorname{lhs}\left(f_{\mathrm{j}}\right)$.

- $f_{l}$ is redundant because $\operatorname{lhs}\left(f_{2}\right) \subseteq \operatorname{lhs}\left(f_{1}\right)^{+}$

- $f_{3}$ is redundant because $\operatorname{lhs}\left(f_{2}\right) \subseteq \operatorname{lhs}\left(f_{3}\right)^{+}$

- $f_{5}$ is redundant because $\operatorname{lhs}\left(f_{4}\right) \subseteq \operatorname{lhs}\left(f_{5}\right)^{+}$

- $f_{8}$ is redundant because $\operatorname{lhs}\left(f_{7}\right) \subseteq \operatorname{lhs}\left(f_{8}\right)^{+}$

- $f_{9}$ is redundant because $\operatorname{lhs}\left(f_{7}\right) \subseteq \operatorname{lhs}\left(f_{9}\right)^{+}$

- $f_{10}$ is redundant because $\operatorname{lhs}\left(f_{11}\right) \subseteq \mathrm{lhs}\left(f_{10}\right)^{+}$

- $f_{13}$ is redundant because $\operatorname{lhs}\left(f_{12}\right) \subseteq \operatorname{lhs}\left(f_{13}\right)^{+}$

- $f_{15}$ is redundant because $\operatorname{lhs}\left(f_{14}\right) \subseteq \operatorname{lhs}\left(f_{15}\right)^{+}$

After removing the redundant dependencies (9), we get the minimal cover $\mathrm{F}_{\mathrm{m}}$ :

$\begin{array}{lll}f_{2}: & \text { clNo,propertyNo } & \rightarrow \text { rentFinish } \\ f_{4}: & \text { clNo } & \rightarrow \text { clName } \\ f_{6}: & \text { clNo,propertyNo } & \rightarrow \text { rentStart } \\ f_{7}: & \text { ownerNo } & \rightarrow \text { oName } \\ f_{11}: & \text { propertyNo } & \rightarrow \text { pAddress } \\ f_{12}: & \text { propertyNo } & \rightarrow \text { rent } \\ f_{14}: \quad \text { propertyNo } & \rightarrow \text { ownerNo } \\ f_{16}: \quad \text { clNo,rentStart } & \rightarrow \text { propertyNo } \\ f_{17}: & \text { propertyNo,rentStart } & \rightarrow \text { clNo }\end{array}$

We have three candidate keys (clNo, propertyNo), (clNo, rentStart) and (propertyNo, rentStart). No matter is the choice of the primary key, we can determine partial and transitive dependencies.

Step 2: We call the Algorithm A4 to generate $F_{f}$ and $F_{p}$ from $F_{m}$. The set of partial dependencies is:

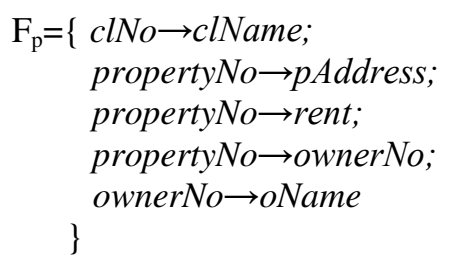

As $\mathrm{F}_{\mathrm{p}} \neq \phi$ the relation ClentRental is not in 2NF. In fact, the attribute clName is partially dependent on the candidate keys (clNo, propertyNo) and (clNo, rentStart). Also, the attributes pAddress, ownerNo and oName are partially dependent on the candidate keys (clNo, propertyNo) and (rentStart, propertyNo).

And the set of full dependencies is:

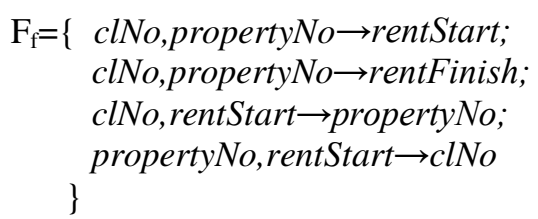

and the set of transitive dependencies is: 


$$
\mathrm{F}_{\mathrm{t}}=\{\text { ownerNo } \rightarrow \text { oName }\}
$$

Step 3: Decomposition of the relation ClientRental into 2NF:

We can choose (clNo,propertyNo) as the primary key, but no special consideration will be given to this key over the other candidate keys. Then the call to the algorithm A5 creates new relations. From $\mathrm{F}_{\mathrm{f}}$, we get the relation

$\mathrm{R}_{(\mathrm{clNo}, \mathrm{propertyNo})}(\underline{\mathrm{clNo}, \text { propertyNo, rentStar, rentFinish) }}$

$\mathrm{R}_{\mathrm{clNo}}$ ( $\left.\underline{\mathrm{clNo}}, \mathrm{clName}\right)$

$\mathrm{R}_{\text {propertyNo }}$ (propertyNo, pAddress, rent,ownerNo, oName)

The key-attributes $c l N o$ and propertyNo cause the violation of $2 \mathrm{NF}$. The three relations are in Second Normal Form as every non-key attribute is fully functionally dependent on the primary key of the relation.

Step 4: Decomposition into 3NF:

As the input of the Algorithm A6 we have $R_{\text {(clNo,propertyNo) }}, R_{\text {clNo }}, R_{\text {propertyNo }}$ and $\mathrm{F}_{\mathrm{t}}$. The only relation that contains the transitive dependency $\mathrm{F}_{\mathrm{t}}$ is $R_{\text {propertyNo. }}$. We then decompose $R_{\text {propertyNo into two }}$ relations and remove the attribute oName from that relation to get all relations into $3 \mathrm{NF}$ :

$\mathrm{R}_{(\mathrm{clNo}, \text { propertyNo) }}$ (clNo,propertyNo, rentStar, rentFinish)

$\mathrm{R}_{\mathrm{clNo}}$ (clNo, clName)

$\mathrm{R}_{\text {propertyNo (propertyNo, pAddress, rent, ownerNo) }}$

$\mathrm{R}_{\text {ownerNo }}$ (ownerNo, oName)

The algorithms are dependency preserving as the original ClientRental relation can be recreated by joining the $3 \mathrm{NF}$ relations $R_{(c I N o, \text { propertyNo) }}, R_{\text {clNo }}, R_{\text {propertyNo }}$ and $R_{\text {ownerNo }}$ through the primary key/foreign key mechanism.

\section{CONCLuSiON}

In this paper we have presented algorithms for relational database normalization into $2 \mathrm{NF}$ and $3 \mathrm{NF}$ using their general definitions in a step-by-step feature. The first step before performing the procedure is to make a preprocessing on the set of dependencies to remove redundant dependencies. We have tested our algorithms on many realistic examples with multiple candidate keys taken from different sources.

This work has mainly the following major advantages: (i) the general and original definition of normal forms is used, (ii) the removal of redundant dependencies, (iii) in all phases, the computation of attributes closure are minimized compared to other algorithms although using a restricted definition of normal forms, and (iv) a primary key is determined for any generated relation. 
International Journal of Database Management Systems ( IJDMS ) Vol.5, No.3, June 2013

\section{REFERENCES}

[1] Thomas, C., Carolyn, B. (2005) Database Systems, A Practical Approach to Design, Implementation, and Management, Pearson Fourth edition .

[2] Bahmani A., Naghibzadeh, M. and Bahmani, B. (2008) "Automatic database normalization and primary key generation", Niagara Falls Canada IEEE.

[3] Beynon-Davies, P. (2004) Database systems, Palgrave Macmillan, Third edition, ISBN 1-40391601-2.

[4] Dongare,Y. V., Dhabe,P. S. and Deshmukh, S. V. (2011) RDBNorma: "A semi-automated tool for relational database schema normalization up to third normal form", International Journal of Database Management Systems, Vol.3, No.1.

[5] Vangipuram, R., Velputa, R., Sravya, V. (2011) "A Web Based Relational database design Tool to Perform Normalization", International Journal of Wisdom Based Computing, Vol.1(3).

[6] Codd, E.F. (1972) "Further normalization of the data base relational model", In Database Systems, Courant Inst. Comptr. Sci. Symp. 6, R. Rustin, Ed., Prentice-Hall, Englewood Cliffs, pp. 33-64.

[7] Elmasri, R., Navathe, S.B. (2003) Fundamentals of Database Systems, Addison Wesley, fourth Edition.

[8] Date, C.J. (2004) Introduction to Database Systems (8th ed.). Boston: Addison-Wesley. ISBN 978-0321-19784-9.

[9] Ullman, J.D. (1982) Principe of Database Systems. Computer Science Press, Rockville, Md.

[10] Maier, D. (1983) The Theory of relational Databases. Computer Science Press, Rockville, Md.

[11] Bernstein, P.A. (1976) "Synthesizing third normal form relations from functional dependencies", ACM Transactions on database Systems, vol.1, No.4, pp.277-298.

[12] Diederich, J., Milton, J. (1988) "New Methods and Fast Algorithms for Database Normalization", ACM Transactions on database Systems, Vol.13, No.3, pp. 339-365. 